\section{Scanning Probe Microscopy in Materials Science}

\author{
Ernst Meyer, Suzanne P. Jarvis, and \\ Nicholas D. Spencer, Guest Editors
}

\begin{abstract}
This brief article introduces the July 2004 issue of MRS Bulletin, focusing on Scanning Probe Microscopy in Materials Science. Those application areas of scanning probe microscopy (SPM) in which the most impact has been made in recent years are covered in the articles in this theme. They include polymers and semiconductors, where scanning force microscopy is now virtually a standard characterization method; magnetism, where magnetic force microscopy has served both as a routine analytical approach and a method for fundamental studies; tribology, where friction force microscopy has opened entirely new vistas of investigation; biological materials, where atomic force microscopy in an aqueous environment allows biosystems to be imaged and measured in a native (or near-native) state; and nanostructured materials, where SPM has often been the only approach capable of elucidating nanostructures.

Keywords: atomic force microscopy, biological materials, magnetism, nanostructured materials, polymers, scanning probe microscopy, scanning tunneling microscopy, semiconductors, tribology.
\end{abstract}

Scanning probe microscopy (SPM) has become a useful tool for characterizing the topography of material surfaces down to the nanometer scale. In that sense, it is a natural-albeit wide-ranging and multifaceted-extension of optical microscopy, ${ }^{1,2}$ and in just a few decades, it has developed into an extremely useful family of techniques (see Table I, on next page). The field of scanning probe microscopy started in 1981 with the invention of scanning tunneling microscopy (STM) by Binnig and Rohrer., The basic components of scanning probe microscopy are shown for the case of STM in Figure 1 . The ability to achieve atomic resolution on the surfaces of metals and semiconductors has turned STM in a short period into an invaluable surface science tool. Together with techniques such as secondaryion mass spectrometry and Auger spectroscopy, STM is a powerful technique for characterizing thin-film growth in molecularbeam epitaxy. Examples include the elucidation of $\mathrm{Si}-\mathrm{Ge}$ heterostructures, which are discussed in the article by Tomitori and Arai in this issue. STM also offers the possibility of scanning tunneling spectroscopy (STS), where valuable information about the local density of states can be gained with far greater spatial resolution than previous apnear-field optical microscopy). proaches using normal and inverse photoemission. Examples include the ability to determine the surface states of semiconductors and the bandgap in superconductors.

One limitation of STM is the necessity for electrical conductivity of the samples. The invention of scanning force microscopy (SFM, or, atomic force microscopy, AFM) by Binnig, Quate, and Gerber in 1986 overcame this limitation, ${ }^{5}$ bringing about a revolution in materials science. The standard resolution of SFM in the repulsive contact mode is in the nanometer range. With the extension of SFM to dynamic force microscopy, it became possible by the mid-1990s to achieve true atomic resolution. ${ }^{6}$ Insulators such as ionic crystals ${ }^{7}$ or oxides ${ }^{8}$ could be imaged on the atomic scale for the first time.

Apart from its impressive performance in high-resolution imaging of insulators, SFM/AFM has the advantage of being applicable in almost any environment, such as air, dry nitrogen, high vacuum, high pressures, or liquids. The last is of critical importance for applications in biology, as the technique allows cells and membranes to be studied in their functional native (wet) environments. AFM of biological samples will be covered by Frederix et al. in this issue.

SFM not only yields maps of sample topography, but also provides information about local properties such as elasticity, charge distributions, magnetization, and chemical reactivity. This distinguishes SFM from other microscopies and makes it an invaluable tool in materials science. Chemical force microscopy ${ }^{9}$ has been used to image the surface chemical state of oxides ${ }^{10}$ and polymers, ${ }^{11}$ and it is now possible to probe chemical forces between a single dangling

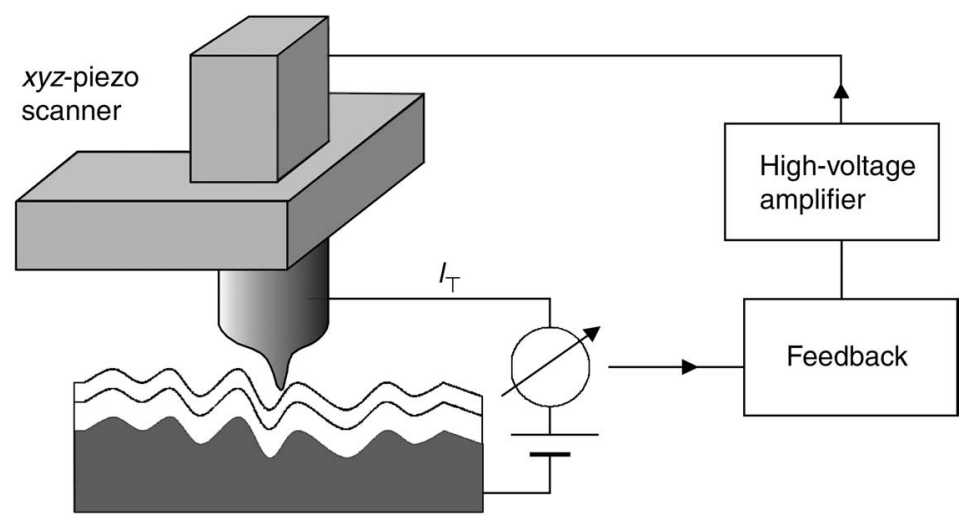

Figure 1. The basic components of scanning probe microscopy (SPM) are shown for the case of scanning tunneling microscopy (STM): xyz-piezo scanners move the probing tip across the sample surface. The tunneling current, $\mathrm{I}_{T}$, is used, in the case of STM, as the input signal to the feedback loop. The output signal of the feedback is amplified and then fed to the z-piezo to control the distance between the probe tip and the sample. Other scanning probe microscopes send different signals to the feedback loop, derived from interactions such as force (scanning force microscopy, magnetic force microscopy) or light (scanning 
Table I: Various Scanning Probe Microscopy (SPM) Techniques.

\begin{tabular}{lll}
\hline Name(s) of Technique & Acronym(s) & \multicolumn{1}{c}{ Mode of Operation } \\
$\begin{array}{l}\text { Scanning tunneling } \\
\text { microscopy }\end{array}$ & STM & Tunneling current controls z-regulating \\
feedback loop.
\end{tabular}

microscopy

feedback loop.

or

Scanning force

microscopy

Friction force

microscopy

or

Lateral force

microscopy

Magnetic force

microscopy

Electric force

microscopy

Scanning Kelvin

probe microscopy

Scanning

capacitance

microscopy

Near-field scanning

optical microscopy

or

Scanning near-field

optical microscopy
Cantilever-spring deflection controls $z$-regulating feedback loop.

FFM, LFM

MFM

EFM

SKPM

SCM

AFM, SFM

Cantilever-spring deflection controls $z$-regulating feedback loop while torsional deflection of spring is displayed.

Deflection of cantilever spring caused by magnetic forces between magnetized tip and surface controls $z$-regulating feedback loop.

Deflection of cantilever spring caused by electrostatic forces between tip and surface controls $z$-regulating feedback loop.

Capacitive force is measured between oscillating tip and surfaces while the sample voltage is varied until the electrostatic field is compensated.

Capacitance is measured between oscillating tip and surfaces while scanning a biased tip above the sample surface.

An optical fiber with a small aperture is scanned in very close proximity to the sample, and the transmitted or reflected light is detected and/or analyzed spectroscopically. bond and atoms in a silicon surface. ${ }^{12}$ Therefore, maps of chemical reactivity can be prepared, opening new perspectives for materials synthesis and catalysis studies. The use of SFM to measure the mechanical, structural, and thermal properties of polymers has also become widespread and is now facilitated by the ability to heat samples in situ in the microscope. The wide range of approaches used in industrial polymer characterization is discussed in the article by Bar and Meyers.

SFM can also be modified to measure the normal and lateral forces between the probe tip and sample simultaneously. This approach is known as friction force microscopy (FFM), or lateral force microscopy (LFM), and it has opened a new window onto the entire field of nanotribology. In 1986, Mate et al. demonstrated atomicscale stick-slip between a tungsten tip and a graphite surface. ${ }^{13}$ Further experiments helped to increase our understanding of the origins of friction. ${ }^{14}$ Recently, the transition from stick-slip to an ultralow friction state has been observed in the laboratory. ${ }^{15}$ Measurements of friction using SPM are described in the article by Perry.
Atomic-scale imaging of morphology (indirectly), or location of orbitals at particular energy levels, When tunneling voltage is varied, the measured current yields a spectrum. This variation of the technique is called scanning tunneling spectroscopy (STS) and yields information on both filled and empty states of the sample's band structure.

Nanoscale measurements of surface morphology, materials properties, and forces between tip (which may be functionalized) and surface.

Friction can be measured and differentiated on a nanometer scale. When this is related to the surface chemistry, this is often referred to as chemical force microscopy (CFM), and tips are often surface-treated in order to enhance contrast.

Magnetic field gradient above a sample.

Electric field gradient or surface potential above a sample.

Map of surface contact potential difference.

Map of surface capacitance.

Optical images of a surface with resolution of $\sim 100 \mathrm{~nm}$; optical images of smaller emitting species, such as single fluorescent molecules.

Many important material properties, such as structural anisotropy, can be elucidated by dynamic modes of AFM. These modes, including dynamic lateral force microscopy, are discussed in the article by Carpick and Eriksson. Magnetic force microscopy (MFM) is an extension of SFM, where magnetic tips are scanned over ferromagnetic samples. This has become an important tool for quality control in magnetic media and is also a key approach to a number of fundamental studies of magnetism, such as imaging magnetic vortices in superconductors and investigating colossal magnetoresistance in 
materials. The characterization of nanomagnets by MFM is discussed by Zhu and Grütter.

In just a few decades, scanning probe microscopy has become a ubiquitous and essential tool in materials imaging and characterization. We hope that the accompanying articles in this issue provide both food for thought and serve as inspiration for further applications of this extraordinarily useful family of techniques.

\section{References}

1. E. Meyer, H.-J. Hug, and R. Bennewitz, Scanning Probe Microscopy: The Lab on a Tip (SpringerVerlag, Berlin, 2003).
2. B. Bhushan, ed., Springer Handbook on Nanotechnology (Springer-Verlag, Berlin, 2003).

3. G. Binnig and H. Rohrer, Helv. Phys. Acta 55 (1982) p. 726

4. G. Binnig, H. Rohrer, Ch. Gerber, and E. Weibel Phys. Rev. Lett. 50 (1983) p. 120

5. G. Binnig, C.F. Quate, and Ch. Gerber, Phys. Rev. Lett. 56 (1986) p. 930.

6. F.J. Giessibl, Science 267 (1995) p. 1451.

7. M. Bammerlin, R. Lüthi, E. Meyer, A. Baratoff, J. Lü, M. Guggisberg, Ch. Gerber, L. Howald, and H.-J. Güntherodt., Probe Microsc. 1 (1997) p. 3 .

8. C. Barth and M. Reichling, Nature 414 (2001) p. 54 .

9. C.D. Frisbie, L.F. Rozsnyai, A. Noy, M.S Wrighton, and C.M. Lieber, Science 265 (1994) p. 2071.
10. G. Hähner, A. Marti, and N.D. Spencer, Tribol. Lett. 3 (1997) p. 359

11. K. Feldman, T. Tervoort, P. Smith, and N.D. Spencer, Langmuir 14 (1998) p. 372.

12. M.A. Lantz, H.J. Hug, P.J.A. van Schendel R. Hoffmann, P. Kappenberger, S. Martin, A Baratoff, and H.-J. Güntherodt, Science 291 (2001) p. 2580.

13. M. Mate, G.M. McClelland, R. Erlandsson, and S. Chiang, Phys. Rev. Lett. 59 (1987) p. 1942.

14. E. Gnecco, R. Bennewitz, T. Gyalog, Ch Loppacher, M. Bammerlin, E. Meyer, and H.-J. Güntherodt., Phys. Rev. Lett. 84 (2000) p. 1172.

15. A. Socoliuc, R. Bennewitz, T. Gyalog, Ch Loppacher, M. Bammerlin, E. Meyer, and H.-J. Güntherodt, Phys. Rev. Lett. 92, 134301 (2004).

\section{Ernst Meyer, Guest Editor} for this issue of $M R S$ Bulletin, is a professor of physics at the University of Basel, Switzerland. His research interests center on the development of surface science techniques such as friction force microscopy and dynamic force microscopy with true atomic resolution. $\mathrm{He}$ is also active in the field of sensors based on micromechanics and magnetic spin resonance detection with force microscopy. Meyer received his $\mathrm{PhD}$ degree at the University of Basel in 1990; his thesis topic was force microscopy on ionic crystals and layered materials. He worked at the IBM Research Center Zurich from 1992 to 1994. In 1997, he joined the faculty at the University of Basel.

Meyer can be reached at the University of Basel, Dept. of Physics and Astronomy, $\mathrm{CH}$ 4056 Basel, Switzerland; tel. 41-61-267-3724, fax 41-61-267-3795, and e-mail ernst.meyer@ unibas.ch.

Suzanne P. Jarvis, Guest Editor for this issue of MRS Bulletin, is a Science Foundation Ireland Principal Investigator at Trinity College Dublin in Ireland. Her research is focused on the use of atomic force microscopy techniques to investigate nanometer-scale functionality in organic and biological molecules. One of her major goals is to make a conceptual advance in understanding the role of water in biological function.

Jarvis graduated from the University of Oxford with a BA degree in physics and a Kodaksponsored DPhil degree in materials. This was followed by postdoctoral fellowships in Japan at the Joint Research Center for Atom Technology, Tsukuba, before she moved on to a permanent post at the Nanotechnology Research Institute, also in Tsukuba. She is a cofounder of the International Nanotribology Forum and has a number of international collaborations supported by FOM; ETH; the Australian Department of Education, Science, and Technology; and the Human Frontier Science Program. Jarvis has made invited contributions to Nanosurface Chemistry and the Encyclopedia of Chemical Physics and Physical Chemistry.

Jarvis can be reached at Trinity College, SFI

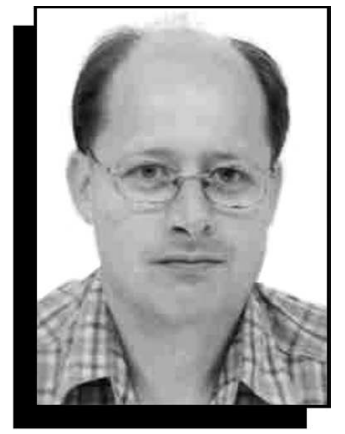

Ernst Meyer

Physics Dept., Dublin 2, Ireland; tel. 353-1-6083026, fax 353-1-608-3027, e-mail suzi.jarvis@tcd.ie, and URL

www.nanofunction.org.

Nicholas D. Spencer, Guest Editor for this issue of MRS Bulletin, has been a professor of surface science and technology at the Swiss Federal Institute of

Technology (ETH

Zurich) since 1993; for the past two years, he has been chair of the Department of Materials, a post he also held from 1996 to 1998 . The principal areas of his research are tribology, biocompatibility, and surface modification and analysis. Atomic force microscopy and the surface forces apparatus play important roles in his group, as well as imaging versions of more traditional surface analytical methods,

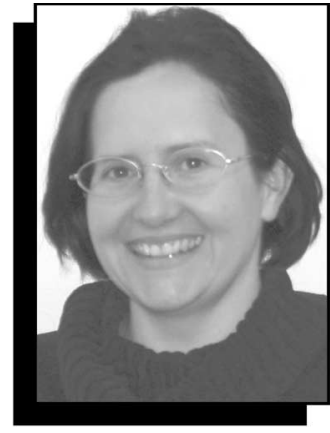

Suzanne P. Jarvis

such as x-ray photoelectron spectroscopy and time-of-flight secondary ion mass spectroscopy. Recent projects include the preparation of chemical gradients, biomimetic lubrication, and high-throughput methods of materials evaluation.

Spencer studied at Cambridge University, obtaining his $\mathrm{PhD}$ degree in 1980 from the Department of Physical Chemistry in the area of gold and silver surface chemistry. Subsequently, he spent two years at the University of California, Berkeley, where he investigated ammonia synthesis on iron single crystals. From 1982 until 1993, he worked for W.R. Grace \& Co. in the areas of catalysis, hightemperature superconductors, surface analysis, and vibrational spectroscopy. He was

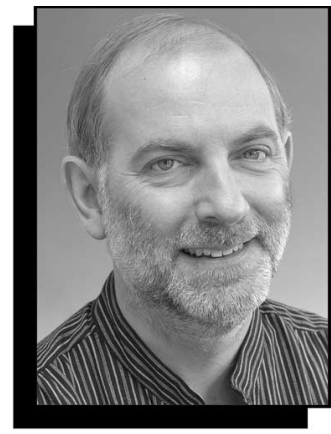

Nicholas D. Spencer

also active as an adjunct professor at the University of Maryland during this time.

$\mathrm{He}$ is editor in chief of Tribology Letters, co-editor of IOP's Chemical Physics series, a member of the editorial boards of Tribology International and Lubrication Science, and co-founder of the International Nanotribology Forum.

Spencer can be reached at the Swiss Federal Institute of Technology, Laboratory for Surface Science and Technology, Dept. of Materials, Zurich, Switzerland; tel. 41-1-632-5850, fax 41-1-633-1027, and e-mail nicholas.spencer@ mat.ethz.ch.

Toyoko Arai is an associate of the School of Materials Science at the Japan Advanced Institute of Science and Technology (JAIST) and a 
project researcher for PRESTO (Precursory Research for Embryonic Science and Technology) at the Japan Science and Technology Agency (JST). Her research interest is in atomic-scale force spectroscopy using scanning probe microscopes. After receiving her MS degree from the Tokyo Institute of Technology (TIT), she joined NEC Corporation in 1987, studying microtribology for magnetic discs. She was a researcher at Kanagawa Academy of Science and Technology from 1992 to 1993. In 1996, she completed her PhD thesis at TIT on the study of electrical double-layer structures at solid-liquid interfaces by atomic force microscopy.

Arai can be reached at JAIST, 1-1 Asahidai, Tatsunokuchi, Ishikawa 923-1292, Japan; tel. 81761-51-1503, fax 81-76151-1149, and e-mail toyoko@jaist.ac.jp.

Georg K. Bar is a senior researcher at Dow

Benelux BV in the

Netherlands, where he is involved in research, development, and characterization of polymer materials. Bar obtained an MS degree in physics (1989) and a PhD degree in chemistry (1992) from the University of Freiburg, Germany. His postdoctoral research at Los Alamos National Laboratory was on structure-property relationships in lowdimensional materials, self-assembled monolayers, and nanostructured surfaces. In 1995, he was appointed group leader of the Surfaces/ Interfaces Group at the Materials Research Center in Freiburg, studying fundamental tip-sample interactions in SPM, as

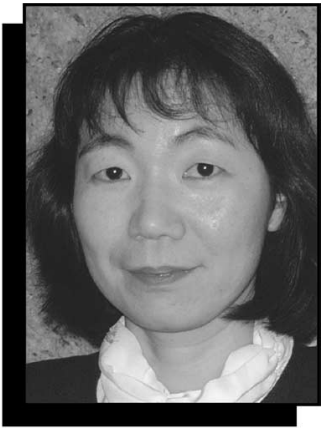

Toyoko Arai

well as structure-property relationships and nanocharacterization of polymer blends, elastomers, block copolymers, composites, latex and thin films. He finished his habilitation in macromolecular chemistry in 2000 and also joined Dow that year.

Bar was co-organizer of the Surface Regio II Symposium '99; the same year, he also received the Eugen Grätz Award for outstanding scientific achievement.

$\mathrm{He}$ is the author or co-author of more than 60 papers.

Bar can be reached at Dow Benelux BV, Analytical Sciences Dept., 4530 AA, Terneuzen, The Netherlands; tel. 31115-67-2302, fax 31-11567-3729, and e-mail gkbar@dow.com.

Robert W. Carpick is an assistant professor in the Engineering Physics Department at the University of Wisconsin, Madison, which he joined in 2000. He carries out research in the areas of nanotribology, nanomechanics, nanostructured materials, and scanning probe microscopy development.

Carpick obtained his BSc degree in physics from the University of Toronto in 1991 and his PhD degree in physics from the University of California, Berkeley, in

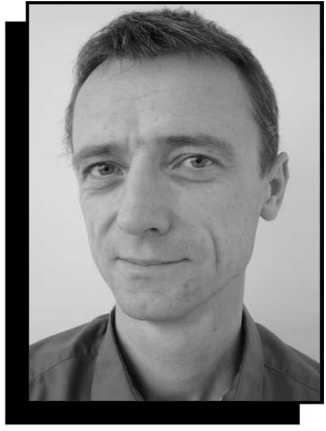

Georg K. Bar

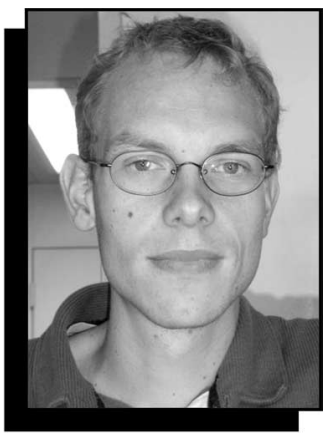

Bart W. Hoogenboom

1997. He then spent two years as a postdoctoral appointee at Sandia National Laboratories in the Surface and Interface Sciences Department and the Biomolecular Materials and Interfaces Department. He has presented short courses on the topics of nanomechanics and nanotribology.

Carpick serves on the editorial board of Review of Scientific Instruments. In 2003, he was awarded an Outstanding New Me-

chanics Educator Award by the American Society for Engineering Education. In 2002, he received a Career Award from the National Science Foundation, Division of Civil and Mechanical Systems.

Carpick can be reached at the University of Wisconsin, Madison, Engineering Physics

Dept., 1500 Engineering Drive., Madison, WI 53706, USA; tel. 608-2634897, fax 608-263-7451,

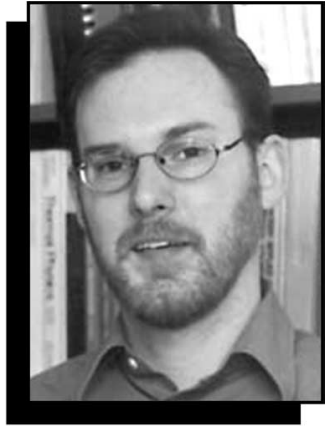

Robert W. Carpick

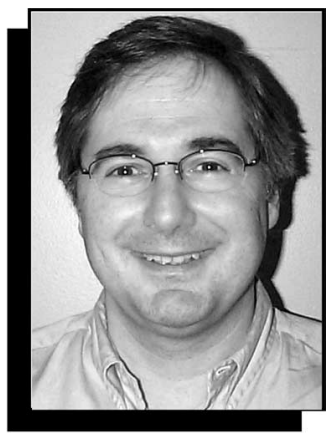

Gregory F. Meyers

and e-mail carpick@ engr.wisc.edu.

Andreas Engel is a professor of structural biology at the Maurice E. Müller Institute for Microscopy at the Biozentrum, University of Basel, Switzerland. After receiving a $\mathrm{PhD}$ degree in physics and doing postdoctoral work in the Biophysics Department at the Johns Hopkins University, he joined the Biozentrum, where he is working on state-of-theart microscopy techniques and developing methods and tools to study the structure, function, and dynamics of membrane proteins in their native environments.

Engel can be reached at the M.E. Müller Institute for Microscopy, Biozentrum, University of Basel, Klingelbergstrasse 70, $\mathrm{CH}-4056$ Basel, Switzerland; tel. 41-61-267-2262, fax 4161-267-2109, and e-mail andreas.engel@unibas.ch.

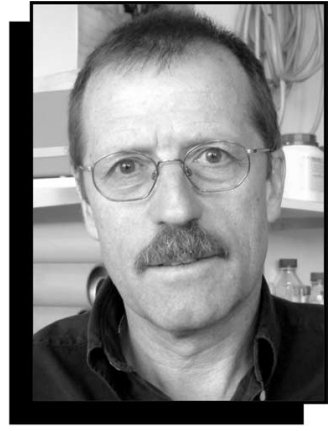

Andreas Engel

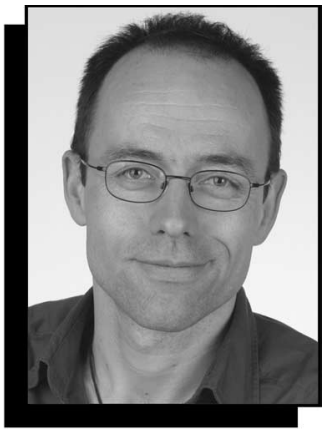

Daniel J. Müller

Mark A. Eriksson is an assistant professor of physics at the University of Wisconsin, Madison. He received his $\mathrm{PhD}$ degree from Harvard University in 1997, where he used cryogenic scanning probe methods to study subsurface nanostructures in GaAs. He was a postdoctoral research fellow at Bell Labs from 1997 to 1999 , where he studied ultralowdensity two-dimensional electron systems. His research interests are in the areas of nanostructures, quantum computation, spins in semiconductors, and scanning probe methods, including intermittent contact atomic force microscopy and electric force microscopy.

Eriksson can be reached at the University of Wisconsin, Madison, Department of Physics, 1150 University Ave., Madison, WI 53706, USA; tel. 608-263-6289, fax 608-265-2334, and e-mail maeriksson@wisc.edu. 


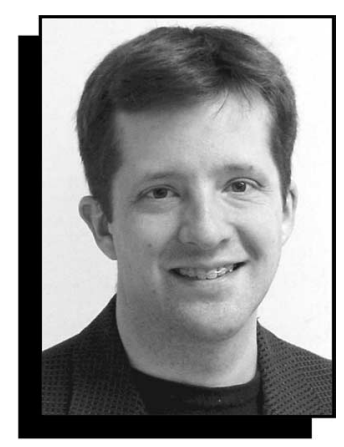

Mark A. Eriksson

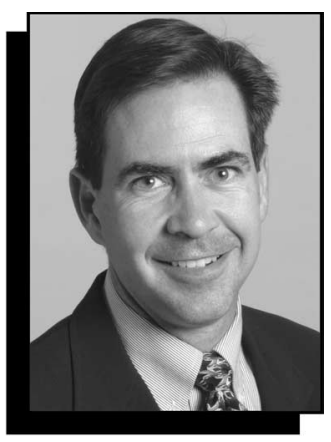

Scott S. Perry

Dimitrios Fotiadis is a postdoctoral researcher at the Maurice E. Müller Institute for Microscopy at the Biozentrum, University of Basel, Switzerland. His research interests include protein chemistry, functional reconstitution, and twodimensional crystallization of membrane proteins, and the determination of their structure using electron and atomic force microscopy. $\mathrm{He}$ received his $\mathrm{PhD}$ degree in biochemistry from the University of Basel in 2000.

Fotiadis can be reached at the M.E. Müller Institute for Microscopy, Biozentrum, University of Basel, Klingelbergstrasse 70, $\mathrm{CH}-4056$ Basel, Switzerland; tel. 41-61-267-0949 and e-mail dimitrios.fotiadis@ unibas.ch.

Patrick Frederix is a postdoctoral researcher at the Maurice E. Müller Institute for Microscopy

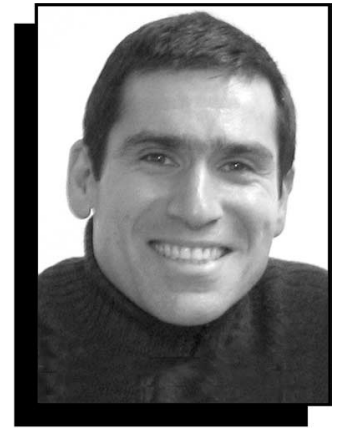

Dimitrios Fotiadis

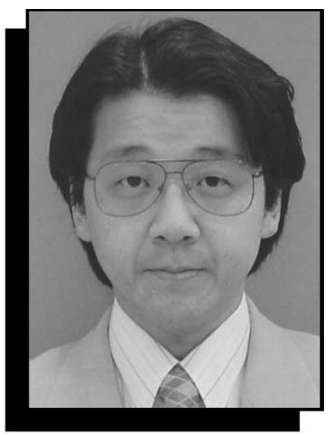

Masahiko Tomitori

at the Biozentrum, University of Basel, Switzerland. He performs research there within the framework of the Swiss National Center of Competence in Research for Nanoscale Sciences, focusing on the development and implementation of multifunctional cantilevers for structural biology. This project is in collaboration with the group of Urs Staufer of the Institute of Microtechnology at the University of Neuchâtel, Switzerland. Frederix has studied applied physics and obtained his PhD degree on fluorescence spectroscopy at Utrecht University.

Frederix can be reached at the M.E. Müller Institute for Microscopy, Biozentrum, University of Basel, Klingelbergstrasse 70, CH-4056 Basel, Switzerland; tel. 41-61-267-2262 and e-mail patrick.frederix@ unibas.ch.

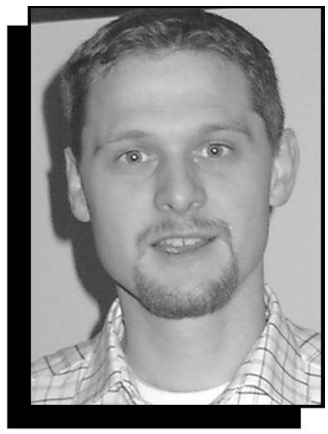

\section{Patrick Frederix}

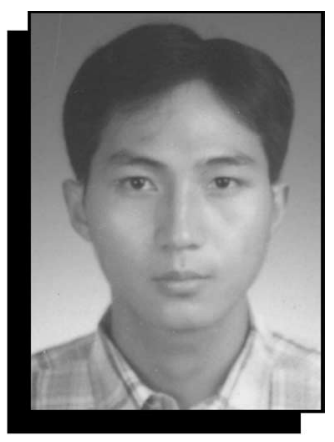

Xiaobin Zhu

Peter Grütter is an associate professor in the Physics Department at McGill University in Montreal. He is the research director of the newly established NSERC NanoInnovation Platform at McGill and is also responsible for the McGill Nanotools Facility. He was recently named director of the Nanoelectronics and Photonics program of the Canadian Institute for Advanced Research.

His group at McGill works on developing tools based primarily on scanning probe microscopy and applying them to problems in nanoscience, in particular, nanoelectronics. They investigate magnetic particles, quantum dots, molecules, and neurons to understand their properties and structure-function relationships.

Grütter did his $\mathrm{PhD}$ work at the University in Basel from 1986 to 1989

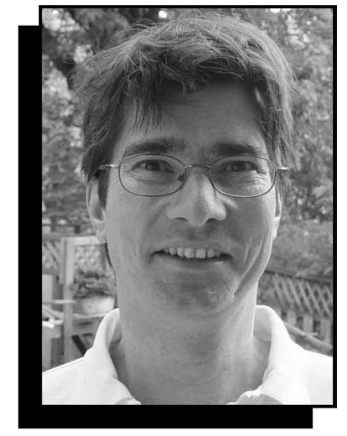

\section{Peter Grütter}

with H.-J. Guntherodt in the field of magnetic force microscopy. After stays at IBM Almaden Research Center and IBM Zurich Research Labs, he joined the faculty of McGill University in 1994.

He has been awarded the NSERC E.W.R.

Staecie Award (2001) and the CIAR Young Explorer Prize ("Top 20 under 40 in Canada" in 2002) and was appointed a William Dawson Scholar by McGill University in 2001 . He is a member of the scientific committee of NanoQuebec, an associate member of CRCN (Centre de recherche sur le cerveau, le comportement, et la neuropsychiatrie) and serves on the board of directors of Quebec Strategic Regroupment in Advanced Materials.

Grütter can be reached at McGill University, Physics Dept., 3600 University St., Montreal, Quebec H3A 2T8, Canada; tel. 514-398-2567, fax 514398-8434, e-mail grutter@ physics.mcgill.ca, and URL www.physics. mcgill.ca/ peter.

Bart W. Hoogenboom is a postdoctoral researcher in biophysics at the Maurice E. Müller Institute for Microscopy at the Biozentrum, University of Basel, Switzerland. His current research fo- cuses on atomic force microscopy with ultrasmall cantilevers. He obtained his $\mathrm{PhD}$ degree (scanning tunneling spectroscopy on superconductors) at the University of Geneva in 2002

Hoogenboom can be reached at the M.E. Müller Institute for Microscopy, Biozentrum, University of Basel, Klingelbergstrasse 70, CH-4056 Basel, Switzerland; tel. 41-61-267-3770 and e-mail bart.hoogenboom@unibas.ch.

Gregory F. Meyers joined the Dow Chemical Company in Midland, Mich., in 1984. For five years, he worked in the area of surface analysis and microscopy, focusing on x-ray photoelectron spectroscopy, scanning Auger microscopy, and scanning electron microscopy techniques in Dow's Corporate Analytical Sciences Laboratory. He began working on scanning probe microscopy in 1989, first with scanning tunneling microscopy and later incorporating atomic force microscopy, tappingmode AFM, nanoindentation, and scanning thermal microscopy. Meyers now oversees a global SPM capability within Dow that includes five sites. He is actively involved in the ASTM E.42.14 Committee on STM/AFM. His current focus is on surface and interfacial characterization for polymer thin films and coatings used for electronic applications.

Meyers obtained a BA degree in chemistry from Bowdoin College in 1979 and a PhD degree in inorganic chemistry from Texas A\&M University in 1985 . He is the author or co-author of 30 publications. 
Meyers can be reached at Dow Chemical Company, 1897E Building, Midland, MI 48667, USA; tel. 989-636-4149, fax 989-638-6443, and e-mail gfmeyers@dow.com.

Daniel J. Müller is a professor and director of the Biotechnological Center at the University of Technology Dresden in Germany. He obtained his $\mathrm{PhD}$ degree in biophysics at the University of Basel, Switzerland, in the group of Andreas Engel, and three years later, he habilitated in biophysics at the same university. Before joining TU-Dresden, he was a group leader at the Max Planck Institute for Molecular Cell Biology and Genetics in Dresden.

Müller can be reached at the University of Technology Dresden, Biotechnological Center, c/o Max Planck Institute for Molecular Cell Biology and Genetics, Pfoten- hauerstrasse 108, D-01307 Dresden, Germany; tel. 49-351-210-2586, fax 49351-210-2020, and e-mail mueller@mpi-cbg.de.

Scott S. Perry is a professor of chemistry and chemical engineering at the University of Houston. His current research interests include the tribological properties of thin organic films, the function of biomimetic polymer lubricants, the structure and chemistry

\section{Cost-Effective Portable Spin Coater}
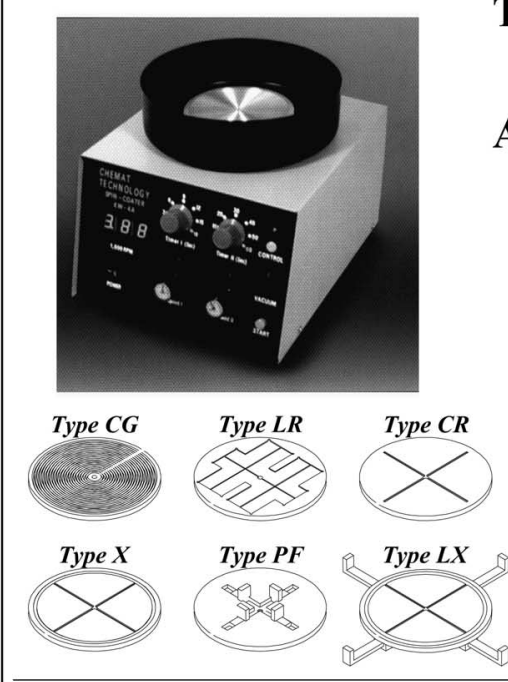

KW-4A SERIES PRODUCT LINE
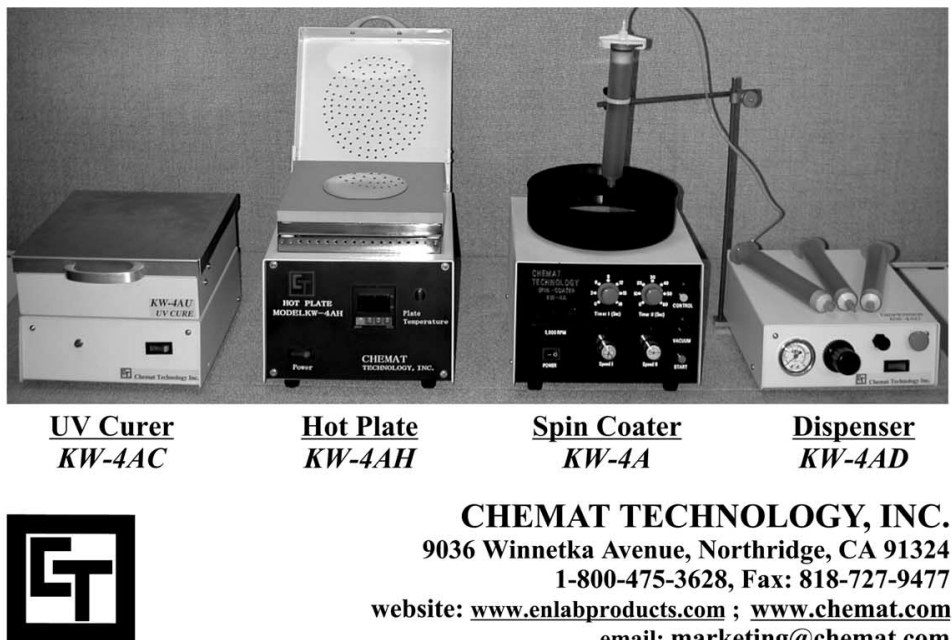

CHEMAT TECHNOLOGY, INC. 9036 Winnetka Avenue, Northridge, CA 91324 1-800-475-3628, Fax: 818-727-9477 website: www.enlabproducts.com ; www.chemat.com email: marketing@chemat.com of chiral metal surfaces, and the chemical and tribological properties of metal carbides. This research entails the use of scanning probe microscopy to map out the nanoscale interfacial properties of these materials and the use of correlated surface analytical probes to assess their chemical and structural nature. Perry is an active participant in the Department of Chemistry at the University of Houston.

He received a BS degree in chemistry from Furman University in 1985 and $\mathrm{PhD}$ degree in chemistry from the University of Texas at Austin in 1991. Afterward, he held a postdoctoral fellowship at the University of California, Berkeley, and was a visiting scientist at the IBM Almaden Research Center. Perry joined the faculty at the University of Houston in 1994.

Perry has received the Cooper Award for Teaching Excellence from the University of Houston (1997), the College of Natural Science and Mathematics Teaching Excellence Award (2000), and the University of Houston Research Excellence Award (2002). In 1999, he received a National Science Foundation Career Award for his research on interfacial properties and his interdisciplinary teaching efforts. In addition to his scholarly work in the fields of surface chemistry and tribology, Perry is co-author with John Hill, Ralph Petrucci, and Terry McCreary of the introductory text General Chemistry.

Perry can be reached at the University of Houston, Center for Materials Chemistry, Houston, TX 77204, USA; tel. 713-743-2715, fax 713-743-2709, and e-mail perry@uh.edu.

Masahiko Tomitori is an associate professor in the School of Materials Science at the Japan Advanced Institute of Science and Technology (JAIST). His research interests are in clarifying various surface and interface phenomena on an atomic scale by means of advanced scanning probe techniques. In 1986, he obtained a DSci degree from the Tokyo Institute of Technology (TIT), focusing on deep levels in II-VI semiconductors. Subsequently, he worked as an associate at TIT, constructing a scanning probe microscope and the related instruments to study nanoscale phenomena in surface science. He joined JAIST in 1994.

Tomitori can be reached at JAIST, 1-1 Asahidai, Tatsunokuchi, Ishikawa 923-1292, Japan; tel. 81-761-51-1501, fax 81-761-51-1149, and e-mail tomitori@jaist.ac.jp.

Xiaobin Zhu is a postdoctoral fellow at the University of Alberta, where he is currently using ultrafast scanning Kerr microscopy and ultrafast scanning tunneling microscopy to study the spin dynamics of magnetic materials. He received a BSc degree from Nanjing University, China, in 1994 and a PhD degree from McGill University, Montreal, in 2002, both in codensedmatter physics.

Zhu can be reached at 412 Avadh Bhatia

Physics Lab, University of Alberta, Edmonton,

Alberta, T6G 2J1

Canada; tel. 780-492-

4197, fax 780-492-0714, and e-mail xzhu@ phys.ualberta.ca. 